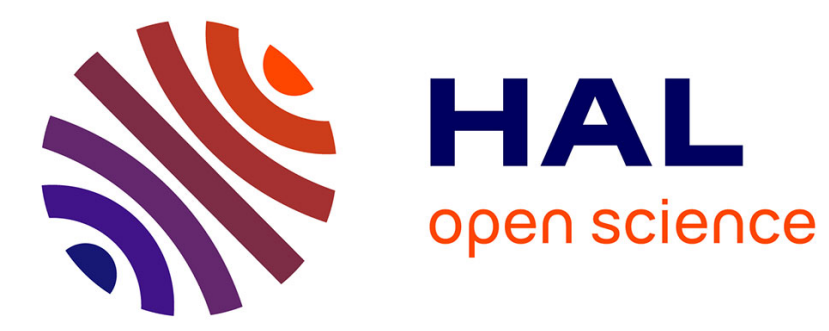

\title{
Comparison of three local frame definitions for the kinematic analysis of the fingers and the wrist
}

Benjamin Goislard de Monsabert, J.M.A. Visser, L. Vigouroux, F.C.T. van Der Helm, H.E.J. Veeger

\section{- To cite this version:}

Benjamin Goislard de Monsabert, J.M.A. Visser, L. Vigouroux, F.C.T. van Der Helm, H.E.J. Veeger. Comparison of three local frame definitions for the kinematic analysis of the fingers and the wrist. Journal of Biomechanics, 2014, 47 (11), pp.2590-2597. 10.1016/j.jbiomech.2014.05.025 . hal-02273334

\section{HAL Id: hal-02273334 \\ https://hal.science/hal-02273334}

Submitted on 28 Aug 2019

HAL is a multi-disciplinary open access archive for the deposit and dissemination of scientific research documents, whether they are published or not. The documents may come from teaching and research institutions in France or abroad, or from public or private research centers.
L'archive ouverte pluridisciplinaire HAL, est destinée au dépôt et à la diffusion de documents scientifiques de niveau recherche, publiés ou non, émanant des établissements d'enseignement et de recherche français ou étrangers, des laboratoires publics ou privés. 


\section{Author's Accepted Manuscript}

Comparison of three local frame definitions for the kinematic analysis of the fingers and the wrist

B. Goislard de Monsabert, J.M.A. Visser, L. Vigouroux, F.C.T. Van der Helm, H.E.J. Veeger

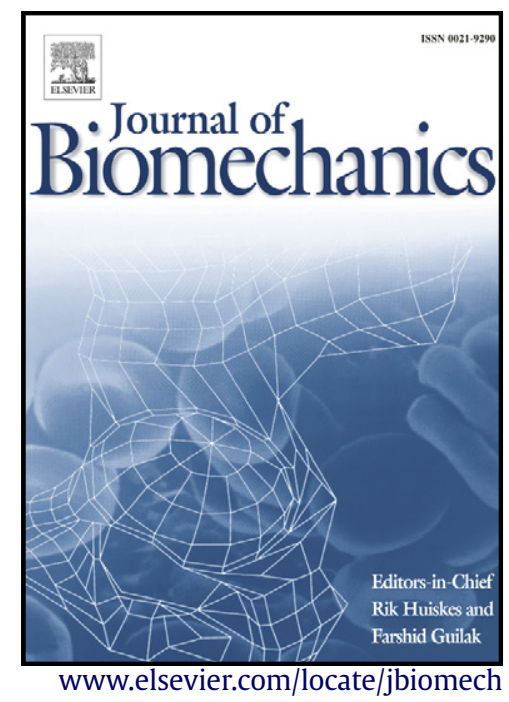

PII: S0021-9290(14)00337-6

DOI: $\quad$ http://dx.doi.org/10.1016/j.jbiomech.2014.05.025

Reference: BM6680

To appear in: Journal of Biomechanics

Accepted date: 30 May 2014

Cite this article as: B. Goislard de Monsabert, J.M.A. Visser, L. Vigouroux, F.C.T. Van der Helm, H.E.J. Veeger, Comparison of three local frame definitions for the kinematic analysis of the fingers and the wrist, Journal of Biomechanics, http: //dx.doi.org/10.1016/j.jbiomech.2014.05.025

This is a PDF file of an unedited manuscript that has been accepted for publication. As a service to our customers we are providing this early version of the manuscript. The manuscript will undergo copyediting, typesetting, and review of the resulting galley proof before it is published in its final citable form. Please note that during the production process errors may be discovered which could affect the content, and all legal disclaimers that apply to the journal pertain. 
Title: Comparison of three local frame definitions for the kinematic analysis of the fingers and the wrist.

Article type: Original Article

\section{Authors:}

Goislard de Monsabert B. ${ }^{1,2}$, Visser J.M.A. ${ }^{2,3}$, Vigouroux L. ${ }^{1}$, Van der Helm F.C.T. ${ }^{2}$, Veeger H.E.J. ${ }^{2,4}$

${ }^{1}$ Aix-Marseille Université, CNRS, ISM UMR 7287, 13288, Marseille cedex 09, France

${ }^{2}$ Department of BioMechanical Engineering, Delft University of Technology, Delft, The Netherlands

${ }^{3}$ Department of Human Kinetic Technology, The Hague University of Applied Sciences, The hague, The Netherlands

${ }^{4}$ Research Institute MOVE, VU Amsterdam, Amsterdam, The Netherlands

\section{Corresponding author:}

Benjamin GOISLARD de MONSABERT

Adress : Institut des Sciences du Mouvement, 163 avenue de Luminy, BP910, 13288

Marseille, cedex 09, FRANCE

Tél: +33491170478

Fax: +33491172252

Email: benjamin.demonsabert@gmail.com

Keywords: hand, kinematics, bony landmarks, instantaneous helical axis, Cardan angles. 


\section{Abstract:}

Because the hand is a complex poly-articular limb, numerous methods have been proposed to investigate its kinematics therefore complicating the comparison between studies and the methodological choices. With the objective of overcoming such issues, the present study compared the effect of three local frame definitions on local axis orientations and joint angles of the fingers and the wrist.

Three local frames were implemented for each segment. The "Reference" frames were aligned with global axes during a static neutral posture. The "Landmark" frames were computed using palpated bony landmarks. The "Functional" frames included a flexionextension axis estimated during functional movements. These definitions were compared with regard to the deviations between obtained local segment axes and the evolution of joint (Cardan) angles during two test motions.

Each definition resulted in specific local frame orientations with deviations of $15^{\circ}$ in average for a given local axis. Interestingly, these deviations produced only slight differences (below $7^{\circ}$ ) regarding flexion-extension Cardan angles indicating that there is no preferred method when only interested in finger flexion-extension movements. In this case, the Reference method was the easiest to implement, but did not provide physiological results for the thumb. Using the Functional frames reduced the kinematic cross-talk on the secondary and tertiary Cardan angles by up to $20^{\circ}$ indicating that the Functional definition is useful when investigating complex three-dimensional movements. Globally, the Landmark definition provides valuable results and, contrary to the other definitions, is applicable for finger deformities or compromised joint rotations. 


\section{NOTATION}

$B_{0}\left(\boldsymbol{x}_{0}, \boldsymbol{y}_{0}, \mathbf{z}_{0}\right) \quad$ Global frame and associated axes

${ }^{s} B_{d}\left({ }^{s} \boldsymbol{x}_{d},{ }^{s} \boldsymbol{y}_{d},{ }^{s} \mathbf{z}_{d}\right) \quad$ Local frame and associated axes of the s segment obtained using the $\mathrm{d}$ local frame definition. ( $\mathrm{d}$ is " $\mathrm{R}$ " for Reference, "L" for Landmark or " $F$ " for functional)

${ }^{d 1} M_{d 2}$ Transformation matrix between two local frames obtained with different definitions ( $d 1$ and $d 2)$ for a given segment.

${ }^{s 1} T_{s 2}$ Transformation matrix between two segments ( 1 1 and s2) for a given local frame definition.

$R(v, \alpha) \quad$ Elementary rotation matrix for a rotation of $\alpha$ about a $\boldsymbol{v}$ axis.

$\boldsymbol{u}_{(P 1, P 2)}$ Unit vector associated to the vector going from the $\mathrm{P} 1$ to the $\mathrm{P} 2$ points

$\boldsymbol{v}_{\text {IHA }}(t)$ Instantaneous helical axis for a given time sample

$\boldsymbol{v}_{\text {flex }}$ Flexion-extension axis estimated during a functional movement

$\theta_{\text {var }}$ Variation in direction of instantaneous helical axis regarding the final flexion-extension axis.

$\Delta_{\text {tot }}$ Total deviation between the local frames obtained with two different definitions of a given segment.

$\Delta_{x}, \Delta_{y}, \Delta_{z}$

Axis deviations between the local axes obtained with two different definitions of a given segment. 
BM-D-13-01063 - Rev. 2

Manuscript (unmarked copy) - 4 / 22

\section{INTRODUCTION}

Assessment of hand and wrist kinematics is relevant for many domains such as hand rehabilitation (Carpinella et al. 2006; Ellis \& Bruton 2002) and finger musculoskeletal modelling (Goislard de Monsabert et al., 2012; Sancho-Bru et al., 2001). However, because the hand is composed of many relatively small segments and comprises more than 15 joints, the measurement of its kinematics is actually quite challenging. Overall, the hand includes 22 degrees of freedom (DoF). Flexion-extension is the primary DoF for all hand joints and abduction-adduction is also possible for several joints but in smaller amplitudes. For the long fingers (i.e. the index, middle, ring and little fingers), while the distal and proximal interphalangeal joints have only one DoF in flexion-extension, the metacarpophalangeal joint additionally allows abduction-adduction. For the thumb, the interphalangeal and metacarpophalangeal joints only moves in flexion-extension whereas the trapeziometacarpal (TMC) joint additionally allows abduction-adduction. The other finger joint rotations are considered as passive, i.e. not mobilised by muscles. The wrist is generally considered as a two-DoF joint (Buchanan et al., 1993).

Generally, the joint kinematics are deduced from the relative motions between the distal and proximal segments (Cappozzo et al., 2005). To this aim, a local frame should be computed for each segment for which two definitions exist (Kontaxis et al., 2009). i) An anatomical frame represents a local expression of the anatomical reference planes, namely sagittal, frontal and transversal, and is generally defined by bony landmarks (further referred as "Landmark" frames) or aligned with an external frame during a postural pose (further referred as "Reference" frames). ii) A functional frame is specifically intended to describe the kinematics of a joint with one of its axes aligned with a joint functional axis (further referred as "Functional" frames). 
BM-D-13-01063 - Rev. 2

Manuscript (unmarked copy) - 5 / 22

For metacarpals and phalanges, the ISB recommended an anatomical frame with a longitudinal axis defined between the geometrical centres of the head and the base of the segment ( $\mathrm{Wu}$ et al., 2005). However, these points are neither palpable nor easy to estimate experimentally. As a result, alternative local frame definitions are used based on either palpable bony landmarks (Buczek et al., 2011; Metcalf et al., 2008) or functional axes (Cerveri et al., 2005; Miyata et al., 2004; Zhang, 2003). For the TMC joint, the ISB recommended to refer to Cooney et al. (1981) who have expressed the trapezium local frame relatively to the third metacarpal one. Because the trapezium bone does not present palpable landmark, the TMC joint rotations have been either expressed regarding the third metacarpal (Carpinella et al., 2006; Cooney et al., 1981) or investigated through medical imaging of the thumb bones (Cerveri et al., 2008; Cheze et al., 2009). For the radius, the recommendations of the ISB for the elbow joint are generally used (van der Helm and Veeger, 1996; Wu et al., 2005).

Since no consensus exists for the computation of local frames for hand segments, the comparison and integration of results from different investigations of hand kinematics is complicated at least. In addition, all approaches have specific pros and cons that thwart the use of a standard approach. To overcome such issues, the objective of this study was to quantify the effect of the local frame definition for hand segments on local axes' orientation and on estimated joint angles. A complete protocol was conducted, including bony landmarks" palpation and functional movements, to implement three definitions: i) the Reference frame, ii) the Landmark frame (adapted from the ISB recommendations) and iii) the Functional frame. We hypothesise that each of the three definitions will result in specific orientations of the local segment axes and that using the Functional frames will reduce the kinematic cross-talk between the flexion-extension Cardan angles and the other angles. 


\section{MATERIALS AND METHODS}

\section{Experimental set up and protocol}

A right-handed male (age: 31 years, height: $184 \mathrm{~cm}$, hand length: $20.6 \mathrm{~cm}$ ) with no history of trauma or pathologies to the right upper limb participated in the experiments after signing an informed consent. The comparison of the three definitions was based on a same single dataset acquired with this subject. The study of a population sample could have interfered with our objectives because the differences between local frame definitions could differ for each individual which could introduce a sample bias in the interpretation of the results.

Three-dimensional positions of hand and forearm segments were tracked using three Optotrak camera sets (Northern Digital Inc., Canada, nominal accuracy: $0.3 \mathrm{~mm}$ ) and 51 active markers (Figure 1B) with a 25-Hz sampling rate. Each segment was tracked using three markers not located on bony landmarks (Kontaxis et al., 2009). To facilitate marker equipment, markers were glued on small plates, three per plate, which were then fastened on the hand using gel tube bandage for the phalanges, double-sided tape for the first and the third metacarpals, and elastic ribbons for the radius.

First, data were acquired to implement the three definitions. This dataset included the position of bony landmarks (Table 1) palpated with a six-marker pointer (accuracy: $0.4 \mathrm{~mm}$ ) and the positions of the markers during a static reference posture (described below) and functional flexion-extension movements (Table 2). Then, to compare the three definitions in dynamic conditions, the subject performed two test motions: the first consisted in a series of long finger flexion motions (straight fingers to fist posture) and the second to the grasping of a 7-cm diameter field hockey ball. 
BM-D-13-01063 - Rev. 2

Manuscript (unmarked copy) - 7 / 22

\section{Local frame definitions}

Three local frame definitions were implemented for each hand segment (Table 3). In accordance with the terminology Kontaxis et al. (2009), the Reference and the Landmark definitions corresponded to two different implementations of anatomical frames and the Functional definition included a functional joint rotation axis. The axes' names and the sign convention were the same for the three definitions:

- $\quad \boldsymbol{x}$ was the transverse axis and was radially oriented

- $y$ was the longitudinal axis and was proximally oriented

- $\quad \mathbf{z}$ was the sagittal axis and was dorsally oriented.

These definitions were different from the ISB recommendations and were chosen for the estimation of Cardan angles. The sequence chosen for Cardan angles decomposition was flexion-abduction-pronation ( $\boldsymbol{x}-\mathbf{z}^{\prime}-\boldsymbol{y}^{\prime \prime}$ in this case) as proposed by An et al. (1979) and usually used for finger musculoskeletal modelling. The $x$ axis corresponded to the flexion-extension movements and the axes' orientations were such that flexion, abduction and pronation represented positive angles.

\section{Reference frame}

The Reference frames corresponded to anatomical axes which were aligned with the laboratory global frame during the static reference posture (Table 3). To help the subject in positioning his hand with regard to the global frame, a "reference board" was specially designed (Figure 1). In particular, the calibration of the optoelectronic system was adapted so that the reference board was aligned with the laboratory global frame and defined a global $x_{0^{-}}$ $\boldsymbol{y}_{0}$ plane with an ascendant $\mathbf{z}_{0}$ axis. The static reference posture corresponded to neutral joint positions as described in the ISB recommendations and was such that the third metacarpal was aligned with the long axis of the forearm, the long fingers' phalanges were aligned with each other and also with the long axis of the forearm and the thumb phalanges were aligned 
BM-D-13-01063 - Rev. 2

Manuscript (unmarked copy) - 8 / 22

with each other. Since the TMC joint rotations cannot be visually assessed, it was decided that the thumb should be in the palm plane and oriented at $35^{\circ}$ of abduction of the third metacarpal.

\section{Landmark frame}

The Landmark frames corresponded to anatomical axes which were implemented using bony landmarks' position and global axes (Table 3). Overall, 30 bony landmarks were palpated on the hand and forearm segments (Table 1) to define local frames. The implementation of this definition (presented in Table 3) was adapted from the propositions of the ISB. For the radius, the landmarks and the local frame computation were identical to those proposed by the ISB for the elbow motions (Wu et al., 2005). The local frame of the trapezium bone was expressed regarding to the third metacarpal using this transformation matrix:

$$
{ }^{T R A} T_{M C 3}=R_{\left(x, 46^{\circ}\right)} \times R_{\left(z, 35^{\circ}\right)} \times R_{\left(y, 82^{\circ}\right)}[1]
$$

\section{Functional frame}

The Functional frames were implemented using functional flexion-extension axes and global axes (Table 3). The flexion-extension axes of all considered hand joints were estimated from the relative orientation of proximal and distal marker clusters during the functional movements (Table 2). The functional axis $\left(\boldsymbol{v}_{\text {func }}\right)$ was the optimal direction vector computed from the instantaneous helical axes (IHA) obtained during the calibration movement (Veeger et al., 1997; Woltring, 1990). For the TMC joint, the clusters of the third and the first metacarpals were used. Contrary to the Landmark definition, the flexion-extension $(\boldsymbol{x})$ axis was computed first and was directly the $\boldsymbol{v}_{\text {func }}$ vector.

To assess the accuracy of functional axes' direction, the angle between the final functional axis $\left(\boldsymbol{v}_{\text {flex }}\right)$ and an IHA $\left(\boldsymbol{v}_{I H A}(t)\right)$ of a given joint was computed for each sample and averaged: 
BM-D-13-01063 - Rev. 2

Manuscript (unmarked copy) - 9 / 22

$$
\theta_{\mathrm{var}}=\frac{1}{T} \sum_{t=1}^{T} \arccos \left(\boldsymbol{v}_{\text {flex }} \cdot \boldsymbol{v}_{\text {IHA }}(t)\right)[2]
$$

\section{Deviations between two definitions}

For each segment, the three definitions were compared regarding the deviations between obtained local segment axes. These deviations were characterized by two variables. First, a total deviation value $\left(\Delta_{\text {tot }}\right)$ between two local frame definitions was computed for each segment using the axis-angle representation (de Vries et al., 2010). For example, the total deviation between the Reference and the Landmark frame of a given segment is computed as follows:

$$
\Delta_{\text {tot }}=\arccos \left(\frac{\operatorname{trace}\left({ }^{L} M_{R}\right)-1}{2}\right) \text { with }{ }^{L} M_{R}=\left(B_{R}\right)^{-1} \times B_{L}, \text {, [3] }
$$

where the ${ }^{L} M_{R}$ matrix describes the relative orientation of the Reference $\left(B_{R}\right)$ and the Landmark $\left(B_{L}\right)$ local frames.

Then, axis deviations $\left(\Delta_{x}, \Delta_{y}, \Delta_{\mathbf{z}}\right)$ between the local axes obtained with two different definitions were computed for each segment as the smallest angles between two vectors (de Vries et al., 2010). For instance, the three axis deviations between the Reference frame and the Landmark frame of a given segment were implemented as follows:

$$
\begin{aligned}
& \Delta_{x}=\arccos \left(\boldsymbol{x}_{R} \cdot \boldsymbol{x}_{L}\right) \\
& \Delta_{y}=\arccos \left(\boldsymbol{y}_{R} \cdot \boldsymbol{y}_{L}\right) \\
& \Delta_{z}=\arccos \left(\mathbf{z}_{R} \cdot \mathbf{z}_{L}\right)
\end{aligned}
$$

\section{Comparison of estimated joint angles}

The local frame definitions were also compared regarding the amplitudes of the joint angles estimated during the two test motions, i.e. finger-flexion and ball-grasping. The orientation of the local frames obtained with each definition was updated sample-by-sample 
BM-D-13-01063 - Rev. 2

Manuscript (unmarked copy) - $10 / 22$

based on the orientation of the marker clusters. The joint angles were then estimated, sampleby-sample and for each definition, by decomposing the relative orientation of proximal and distal local frames using the $\boldsymbol{x}-\mathbf{z}^{\prime}-\boldsymbol{y}$ '’ sequence of Cardan angles presented above. For each definition, the amplitudes of the three Cardan angles were computed for each joint as the difference between the maximum and the minimum angle values observed over all samples of a given test motion.

\section{RESULTS}

\section{Accuracy of functional axis}

The variation in direction of IHA vectors $\left(\theta_{v a r}\right)$ ranged from $5.3^{\circ}$ for the index finger PIP joint to $23.4^{\circ}$ for the thumb TMC joint with a mean value of $11.04 \pm 4.43^{\circ}$ among all joints. Except for the TMC joint, the $\theta_{v a r}$ value was lower than $15^{\circ}$ for each joint.

\section{Deviations between two definitions}

The deviations between two local frames differently defined varied widely depending on the segment considered (Figure 2 and Table 4). The total deviation ranged from $4.9^{\circ}$ to $36.9^{\circ}$ among segments of the long fingers and the radius, and up to $70.8^{\circ}$ for the thumb. For each segment except those of the thumb, the largest total deviation values were observed between Functional and Landmark frames. In correspondence with total deviation values, the axis deviations varied largely among segments and ranged between $0.1^{\circ}$ to $32.5^{\circ}$ for the long fingers and the radius, and up to $70.2^{\circ}$ for the thumb. On average, the axis deviation $(x, y$ or $z)$ between two definitions was around $15^{\circ}$, except for the thumb, where $60^{\circ}$ deviations were observed. Low deviation values (below $10^{\circ}$ in average) were observed between the $x$ axis of 
BM-D-13-01063 - Rev. 2

Manuscript (unmarked copy) - $11 / 22$

the Landmark and Reference frames and between the $y$ axis of the Functional and Reference frames.

\section{Comparison of estimated joint angles}

The differences between Cardan angles obtained with each definition during the fingerflexion and the ball-grasping test motions are summarized in Table 5 and illustrated in Figure 3 and 4. For the finger-flexion motion, the amplitudes estimated for the flexion-extension were comparable with a maximal difference of $7.0^{\circ}$ between two definitions for a given joint (Table 5). During this motion, the Functional frames resulted in reduced amplitudes for abduction-adduction and pronation-supination with differences with another definition of up to $23.6^{\circ}$ and $18.8^{\circ}$, respectively. During the ball grasping-motion, the long finger joints and the wrist exhibited similar results as during the finger-flexion motion with lower differences between definitions. For the thumb, the Cardan angles estimated with the Reference frames during ball-grasping resulted in higher amplitudes on all three Cardan angles with differences of up to $15^{\circ}$ with other definitions (Figure 4).

\section{DISCUSSION}

Currently, the investigation of the hand kinematics remains challenging and there is neither a consensus on which method should be used nor a clear proposal regarding what could be used (Kontaxis et al. 2009). In this context, it is important to quantify how the choice of a method can influence the kinematic analysis in order to facilitate the comparison and the integration of results from different studies. With this in mind, the present study conducted a complete protocol, including bony landmarks' palpation and functional movements, for the kinematic analysis of hand and wrist joints and compared three existing local frame definitions. 
BM-D-13-01063 - Rev. 2

Manuscript (unmarked copy) - 12 / 22

In agreement with our hypotheses, each definition resulted in specific local frame orientations with deviations between estimated axes of about $15^{\circ}$ on average and up to $23.6^{\circ}$ among long fingers' segments and the radius. Despite these deviations, the three definitions showed only small differences regarding flexion-extension Cardan angles, i.e. below $7^{\circ}$ for all long finger joints. Therefore, for kinematic analyses focusing only on the estimation of flexion-extension for the long fingers regardless of the segment orientations, all three methods thus appear to be as suitable. However, the Functional frame definition exhibited lower amplitudes on the secondary (abduction-adduction) and tertiary (pronation-supination) Cardan angles and differed from other definitions for more than $20^{\circ}$ on several joints. As Piazza and Cavanagh (2000) demonstrated for the knee, using a functional flexion axis tends to reduce the cross-talk between the three Cardan angles, leading to an easier interpretation of the second and third axis in terms of 'real' motion occurring in the joint. However, the larger amplitudes observed for the Reference and Landmark frames were not mechanically incorrect but resulted of different deviations between the joint rotation axes and the local frames axes. The Functional frame method seems therefore easier applicable for the analysis of complex hand movements involving combined joint rotations and/or multiple fingers such as unscrewing a bottle cap or folding a paper (Rácz et al., 2012).

Compared to long fingers, the deviations between the three local frame definitions for the thumb were particularly large, with axis deviations between two definitions reaching $70^{\circ}$. These high values were due to the particular definition of the Reference frame for the thumb which consisted of rotating the global frame at $35^{\circ}$ of abduction. Although the Reference longitudinal (y) axis was similar to the Landmark one (Table 4), the Reference flexion $(\boldsymbol{x})$ and abduction (z) axes were largely deviated from those of the Landmark and the Functional definitions (Figure 2 and Table 4). Because of these high deviations, the Reference frames resulted in not physiologically interpretable Cardan angles for all thumb joints (Figure 4) and 
BM-D-13-01063 - Rev. 2

Manuscript (unmarked copy) - $13 / 22$

are therefore inappropriate to study thumb movements. Therefore, while the Reference frames represent a relatively simple way to investigate finger flexion-extension, when studying hand movements involving the thumb, the Landmark and Functional frames are more appropriate since they provided more physiological results.

The present findings confirmed our hypotheses that each definition results in specific local frame orientations and that the deviations between local axes result in important differences regarding secondary and tertiary Cardan angles. Therefore, care should be taken when choosing a local frame definition and when comparing results of studies using different definitions. For example, segment and joint kinematics are used in inverse musculoskeletal modelling to compute joint torques and muscle moment arms to further estimate muscle forces. In this case, two local frame definitions might therefore result in important differences regarding the estimated muscle load sharing (Valero-Cuevas et al., 2003; Vigouroux et al., 2009). However, the comparison of anatomical (Reference and Landmark definitions) and functional axes did not point out an ideal method for the kinematic analysis of the wrist and fingers. All three methods indeed present specific advantages and drawbacks so that the choice of a definition will depend on the research questions and the protocol possibilities. It should also be noted that, regardless of the chosen definition, care should always be taken when interpreting Cardan angles as neither anatomical nor functional axes represent the "true" axes of rotation of a joint (Piazza and Cavanagh, 2000).

The advantage of the Functional definition was the use of an individualised estimation of the flexion axes which resulted in more physiologically interpretable Cardan angles on the three joint rotations. However, functional axes might be difficult to estimate for persons with compromised joint rotations such as rheumatoid arthritis patients suffering from ulnar deviations (Figure 5A) or boutonniere deformities (Figure 5B). 
BM-D-13-01063 - Rev. 2

Manuscript (unmarked copy) - 14 / 22

If the kinematic analysis only focuses on finger flexion-extension, our results show that all three methods provide satisfying results. In that case, the Reference method is very easy to implement as only a recording of the marker cluster positions in the neutral posture is needed. Such a setup can be interesting when the protocol must be as short as possible, e.g. when pain is involved. However, the Reference frames are not sensitive to anatomical differences. For instance, patients presenting compromised joint postures might not be able to align their segments in the neutral posture so that the Reference definition will poorly represent the bone orientation (Figure 5). For similar reasons, the Reference definition was not adapted for tracking thumb motions (discussed above).

The Landmark definition provides a subject-specific and segment-specific estimation of the segment orientation and, contrary to the two other definitions, can be used despite hand deformities (Figure 5). For these reasons, the use of bony landmarks has been widely recommended as a standard for the kinematic analysis of both the upper (Kontaxis et al., 2009; Wu et al., 2005) and lower (Cappozzo et al., 2005; Wu et al., 2002) extremities. The main drawbacks of this definition are the palpation of numerous bony landmarks and, as with the Reference method, a risk for cross-talk misinterpretation.

Several points should be highlighted when considering the findings of this study. For instance, the skin movement artefacts, reaching up to $10 \mathrm{~mm}$ for finger segments (Ryu et al., 2006), have not been corrected here and might have different effects on each method. The use of plates equipped with markers might also amplify skin movements due to their own inertia (Leardini et al., 2005), although no significant differences between the kinematics estimated with skin-mounted and plate-mounted markers has yet been proved (Nester et al., 2007). Since more than 50 markers were required to track each segment with three markers, further studies could investigate how the number of markers influences the estimation of finger kinematics in order to optimize experimental procedures. It should also be noted that the 
BM-D-13-01063 - Rev. 2

Manuscript (unmarked copy) - 15 / 22

functional flexion-extension movements presented here might have included other joint rotations which could influence the direction of estimated axes. Nevertheless, since "pure" uniaxial motions can neither be actively executed nor controlled by external examination, the functional movements were designed to be relatively intuitive and simple tasks while reducing at best other rotations than flexion-extension.

Elaborating standardised kinematic analysis is an important step to improve our knowledge of hand kinematics. In the long-term, the main goal would be to build reference databases, as it has been done for gait analysis, describing healthy finger free movements or grasping tasks. Before that, the local frame definitions tested here should be validated by comparing the results obtained with each definition using external markers with the real bone movements measured by medical imaging. Overall, designing standardized definitions of local frames should remain a priority since, regardless of the measurement technology employed, their computation will remain a necessary step to track bone movements and estimate joint rotations.

\section{CONFLICT OF INTEREST STATEMENT}

All authors agree that there is no conflict of interests that could inappropriately influence their work.

\section{ACKNOWLEDGMENT}

The present work did not involve any external source of funding. 


\section{REFERENCES}

An, K.N., Chao, E.Y., Cooney III, W.P., Linscheid, R.L., 1979. Normative model of human hand for biomechanical analysis. Journal of Biomechanics 12, 775-788.

Buchanan, T.S., Moniz, M.J., Dewald, J.P., Zev Rymer, W., 1993. Estimation of muscle forces about the wrist joint during isometric tasks using an EMG coefficient method. Journal of biomechanics 26, 547-560.

Buczek, F.L., Sinsel, E.W., Gloekler, D.S., Wimer, B.M., Warren, C.M., Wu, J.Z., 2011. Kinematic performance of a six degree-of-freedom hand model (6DHand) for use in occupational biomechanics. Journal of Biomechanics 44, 1805-1809.

Cappozzo, A., Della Croce, U., Leardini, A., Chiari, L., 2005. Human movement analysis using stereophotogrammetry: Part 1: theoretical background. Gait \& Posture 21, 186196.

Carpinella, I., Mazzoleni, P., Rabuffetti, M., Thorsen, R., Ferrarin, M., 2006. Experimental protocol for the kinematic analysis of the hand: Definition and repeatability. Gait \& Posture 23, 445-454.

Cerveri, P., Lopomo, N., Pedotti, A., Ferrigno, G., 2005. Derivation of centers and axes of rotation for wrist and fingers in a hand kinematic model: methods and reliability results. Annals of Biomedical Engineering 33, 402-412.

Cerveri, P., Momi, E.D., Marchente, M., Lopomo, N., Baud-Bovy, G., Barros, R.M.L., Ferrigno, G., 2008. In Vivo Validation of a Realistic Kinematic Model for the Trapezio-Metacarpal Joint Using an Optoelectronic System. Annals of Biomedical Engineering 36, 1268-1280.

Cheze, L., Dumas, R., Comtet, J.J., Rumelhart, C., Fayet, M., 2009. A joint coordinate system proposal for the study of the trapeziometacarpal joint kinematics. Computer Methods in Biomechanics and Biomedical Engineering 12, 277-282.

Cooney, W., Lucca, M., Chao, E., Linscheid, R., 1981. The kinesiology of the thumb trapeziometacarpal joint. Journal of Bone and Joint Surgery, American Volume 63, 1371-1381.

De Vries, W.H.K., Veeger, H.E.J., Cutti, A.G., Baten, C., van der Helm, F.C.T., 2010. Functionally interpretable local coordinate systems for the upper extremity using inertial \& magnetic measurement systems. Journal of Biomechanics 43, 1983-1988.

Ellis, B., Bruton, A., 2002. A study to compare the reliability of composite finger flexion with goniometry for measurement of range of motion in the hand. Clinical Rehabilitation $16,562-570$.

Goislard de Monsabert, B., Rossi, J., Berton, E., Vigouroux, L., 2012. Quantification of Hand and Forearm Muscle Forces during a Maximal Power Grip Task. Medicine and science in sports and exercise 44, 1906-1916.

Kontaxis, A., Cutti, A.G., Johnson, G.R., Veeger, H.E.J., 2009. A framework for the definition of standardized protocols for measuring upper-extremity kinematics. Clinical Biomechanics 24, 246-253.

Leardini, A., Chiari, L., Croce, U.D., Cappozzo, A., 2005. Human movement analysis using stereophotogrammetry: Part 3. Soft tissue artifact assessment and compensation. Gait \& Posture 21, 212-225.

Metcalf, C.D., Notley, S.V., Chappell, P.H., Burridge, J.H., Yule, V.T., 2008. Validation and application of a computational model for wrist and hand movements using surface markers. IEEE Transactions on Bio-Medical Engineering 55, 1199-1210. 
Miyata, N., Kouchi, M., Kurihara, T., Mochimaru, M., 2004. Modeling of human hand link structure from optical motion capture data, in: 2004 IEEE/RSJ International Conference on Intelligent Robots and Systems, 2004. (IROS 2004). Proceedings. Presented at the $2004 \mathrm{IEEE} / \mathrm{RSJ}$ International Conference on Intelligent Robots and Systems, 2004. (IROS 2004). Proceedings, IEEE, pp. 2129- 2135 vol.3.

Nester, C., Jones, R.K., Liu, A., Howard, D., Lundberg, A., Arndt, A., Lundgren, P., Stacoff, A., Wolf, P., 2007. Foot kinematics during walking measured using bone and surface mounted markers. Journal of Biomechanics 40, 3412-3423.

Piazza, S.J., Cavanagh, P.R., 2000. Measurement of the screw-home motion of the knee is sensitive to errors in axis alignment. Journal of Biomechanics 33, 1029-1034.

Rácz, K., Brown, D., Valero-Cuevas, F.J., 2012. An involuntary stereotypical grasp tendency pervades voluntary dynamic multifinger manipulation. Journal of Neurophysiology 108, 2896-2911.

Ryu, J.H., Miyata, N., Kouchi, M., Mochimaru, M., Lee, K.H., 2006. Analysis of skin movement with respect to flexional bone motion using MR images of a hand. Journal of Biomechanics 39, 844-852.

Sancho-Bru, J.L., Pérez-González, A., Vergara-Monedero, M., Giurintano, D., 2001. A 3-D dynamic model of human finger for studying free movements. Journal of Biomechanics 34, 1491-1500.

Valero-Cuevas, F.J., Johanson, M.E., Towles, J.D., 2003. Towards a realistic biomechanical model of the thumb: the choice of kinematic description may be more critical than the solution method or the variability/uncertainty of musculoskeletal parameters. Journal of Biomechanics 36, 1019-1030.

Van der Helm, F.C.T., Veeger, H.E.J., 1996. Quasi-static analysis of muscle forces in the shoulder mechanism during wheelchair propulsion. Journal of Biomechanics 29, 39 52.

Veeger, H.E.J., Yu, B., An, K.-N., Rozendal, R.H., 1997. Parameters for modeling the upper extremity. Journal of Biomechanics 30, 647-652.

Vigouroux, L., Domalain, M., Berton, E., 2009. Comparison of tendon tensions estimated from two biomechanical models of the thumb. Journal of Biomechanics 42, 17721777.

Woltring, H.J., 1990. Data processing and error analysis., in: Biomechanics ofHuman Movement: Applications in Rehabilitation, Sports and Ergonomics. Bertec Corporation, Worthington, OH, pp. 203-237.

Wu, G., Siegler, S., Allard, P., Kirtley, C., Leardini, A., Rosenbaum, D., Whittle, M., D’Lima, D.D., Cristofolini, L., Witte, H., Schmid, O., Stokes, I., 2002. ISB recommendation on definitions of joint coordinate system of various joints for the reporting of human joint motion - part I: ankle, hip, and spine. Journal of Biomechanics 35, 543-548.

Wu, G., van der Helm, F.C.T., Veeger, H.E.J.D., Makhsous, M., Van Roy, P., Anglin, C., Nagels, J., Karduna, A.R., McQuade, K., Wang, X., Werner, F.W., Buchholz, B., 2005. ISB recommendation on definitions of joint coordinate systems of various joints for the reporting of human joint motion--Part II: shoulder, elbow, wrist and hand. Journal of Biomechanics 38, 981-992.

Zhang, X., 2003. Determining finger segmental centers of rotation in flexion-extension based on surface marker measurement. Journal of Biomechanics 36, 1097-1102. 
BM-D-13-01063 - Rev. 2

Manuscript (unmarked copy) - 18 / 22

Table 1 - Name and location of the palpated bony landmarks.

\begin{tabular}{|c|c|}
\hline Name & Location \\
\hline \multicolumn{2}{|l|}{ Forearm } \\
\hline EL & Most caudal point on lateral epicondyle \\
\hline EM & Most caudal point on medial epicondyle \\
\hline US & Most caudal-medial point on ulnar styloid \\
\hline $\mathrm{RS}$ & Most caudal-lateral point on radial styloid \\
\hline \multicolumn{2}{|l|}{ Fingers } \\
\hline $\mathrm{MCb}$ & Most dorsal point of the metacarpal base \\
\hline $\mathrm{MCh}$ & Most dorsal point the metacarpal head \\
\hline $\mathrm{PPh}$ & Most dorsal point of the proximal phalanx head \\
\hline $\mathrm{MPh}$ & Most dorsal point the medial phalanx head \\
\hline $\mathrm{DPh}$ & Most distal point of the distal phalanx head \\
\hline \multicolumn{2}{|l|}{ Thumb } \\
\hline $\mathrm{MC1b}$ & Most dorsal point of the first metacarpal base \\
\hline $\mathrm{MC} 1 \mathrm{~h}$ & Most dorsal point of the first metacarpal head \\
\hline $\mathrm{MC} 1 \mathrm{u}$ & Most dorsal-ulnar point of the first metacarpal head \\
\hline $\mathrm{MC} 1 \mathrm{r}$ & Most dorsal-radial point of the first metacarpal head \\
\hline PP1h & Most dorsal point of the thumb proximal phalanx head \\
\hline DP1h & Most distal point the thumb proximal phalanx head \\
\hline
\end{tabular}


BM-D-13-01063 - Rev. 2

Manuscript (unmarked copy) - 19/22

Table 2 - Description of the functional movements for the estimation of the flexionextension rotation axes. * indicates that Metacarpophalangeal and interphalangeal joints were moved at the same time.

\begin{tabular}{|c|c|}
\hline Joints & Description of the global posture and the movement executed \\
\hline Wrist & $\begin{array}{l}\text { Forearm mid-pronated lying on a flat surface. The hand is moving } \\
\text { alternatively in the palmar and dorsal direction with the ulnar side sliding on } \\
\text { the surface. }\end{array}$ \\
\hline Fingers* & $\begin{array}{l}\text { Similar posture than for the wrist flexion. } \\
\text { All long fingers are moving back and forth from straight position to closed } \\
\text { fist. }\end{array}$ \\
\hline Thumb* & $\begin{array}{l}\text { Same posture than for the wrist flexion. } \\
\text { At rest, the thumb tip lies on the middle phalanx of the index finger which } \\
\text { joints are slightly flexed. Then, the thumb is moved upward and downward } \\
\text { without deviating from the plane which contained its phalanges at rest. }\end{array}$ \\
\hline TMC joint & $\begin{array}{l}\text { Same posture than for the wrist flexion. } \\
\text { The thumb is moved in a plane as parallel as possible to the plane including } \\
\text { the dorsal aspects of the second and third metacarpals. Subjects must } \\
\text { minimise the thumb pronation-supination rotations. }\end{array}$ \\
\hline
\end{tabular}


BM-D-13-01063 - Rev. 2

Manuscript (unmarked copy) - 20 / 22

Table 3 - Equations used to implement each definition.

\begin{tabular}{llll}
\hline & \multicolumn{3}{c}{ Local frame definition } \\
\hline Radius & Reference (R) & Landmark (L) & Functional (F) \\
$1^{\text {st }}$ axis & $\boldsymbol{x}_{R}=\boldsymbol{x}_{0}$ & $\boldsymbol{y}_{L}=\boldsymbol{u}_{(R S, E L)}$ & $\boldsymbol{x}_{F}=\boldsymbol{v}_{\text {flex }}$ \\
$2^{\text {nd }}$ axis & $\boldsymbol{y}_{R}=\mathbf{z}_{0} \times \boldsymbol{x}_{R}$ & $\mathbf{z}_{L}=\boldsymbol{u}_{(U S, R S)} \times \boldsymbol{y}_{L}$ & $\boldsymbol{y}_{F}=\mathbf{z}_{0} \times \boldsymbol{x}_{F}$ \\
$3^{\text {rd }}$ axis & $\mathbf{z}_{R}=\boldsymbol{x}_{R} \times \boldsymbol{y}_{R}$ & $\boldsymbol{x}_{L}=\boldsymbol{y}_{L} \times \mathbf{z}_{L}$ & $\mathbf{z}_{F}=\boldsymbol{x}_{F} \times \boldsymbol{y}_{F}$
\end{tabular}

Fingers*

$\begin{array}{llll}1^{\text {st }} \text { axis } & \boldsymbol{x}_{R}=\boldsymbol{x}_{0} & \boldsymbol{y}_{L}=\boldsymbol{u}_{(L P, L D)} * * * & \boldsymbol{x}_{F}=\boldsymbol{v}_{\text {flex }} \\ 2^{\text {nd }} \text { axis } & \mathbf{z}_{R}=\mathbf{z}_{0} \times \boldsymbol{x}_{R} & \boldsymbol{x}_{L}=\boldsymbol{y}_{L} \times \mathbf{z}_{0} & \boldsymbol{y}_{F}=\mathbf{z}_{0} \times \boldsymbol{x}_{F} \\ 3^{\text {rd }} \text { axis } & \mathbf{z}_{R}=\boldsymbol{x}_{R} \times \boldsymbol{y}_{R} & \mathbf{z}_{L}=\boldsymbol{x}_{L} \times \boldsymbol{y}_{L} & \mathbf{z}_{F}=\boldsymbol{x}_{F} \times \boldsymbol{y}_{F}\end{array}$

Trapezium

$$
\begin{array}{llll}
1^{\text {st }} \text { axis } & \boldsymbol{x}_{R}=R_{\left(\mathbf{z}, 35^{\circ}\right)} \boldsymbol{x}_{0} & \boldsymbol{y}_{L}={ }^{T R A} T_{M C 3}{ }^{M C 3} \boldsymbol{y}_{L} & \boldsymbol{x}_{F}=\boldsymbol{v}_{\text {flex }} \\
2^{\text {nd }} \text { axis } & \boldsymbol{y}_{R}=\mathbf{z}_{0} \times \boldsymbol{x}_{R} & \boldsymbol{x}_{L}=\boldsymbol{y}_{L} \times\left({ }^{T R A} T_{M C 3}{ }^{M C 3} \boldsymbol{z}_{L}\right) & \boldsymbol{z}_{F}=\boldsymbol{x}_{F} \times \boldsymbol{y}_{R} \\
3^{\text {rd }} \text { axis } & \mathbf{z}_{R}=\boldsymbol{x}_{R} \times \boldsymbol{y}_{R} & \mathbf{z}_{L}=\boldsymbol{x}_{L} \times \boldsymbol{y}_{L} & \boldsymbol{y}_{F}=\mathbf{z}_{F} \times \boldsymbol{x}_{F}
\end{array}
$$

Thumb**

$$
\begin{array}{llll}
1^{\text {st }} \text { axis } & \boldsymbol{x}_{R}=R_{\left(\boldsymbol{z}, 35^{\circ}\right)} \boldsymbol{x}_{0} & \boldsymbol{y}_{L}=\boldsymbol{u}_{(L P, L D)} & \boldsymbol{x}_{F}=\boldsymbol{v}_{\text {flex }} \\
2^{\text {nd }} \text { axis } & \boldsymbol{y}_{R}=\mathbf{z}_{0} \times \boldsymbol{x}_{R} & \boldsymbol{z}_{L}=\boldsymbol{u}_{(M P R, M P U)} \times \boldsymbol{y}_{L} & \boldsymbol{z}_{F}=\boldsymbol{x}_{F} \times \boldsymbol{y}_{R} \\
3^{\text {rd }} \text { axis } & \boldsymbol{z}_{R}=\boldsymbol{x}_{R} \times \boldsymbol{y}_{R} & \boldsymbol{x}_{L}=\boldsymbol{y}_{L} \times \mathbf{z}_{L} & \boldsymbol{y}_{F}=\boldsymbol{z}_{F} \times \boldsymbol{x}_{F}
\end{array}
$$

*: "Fingers" refers to the $2^{\text {nd }}$ to $5^{\text {th }}$ metacarpals and the phalanges of the long fingers

**: "Thumb" refers to the first metacarpal and the phalanges of the thumb

***: LP and LD refer to the landmarks representing the proximal and distal ends of the segment, respectively.

Other notations are defined in the text or in the Nomenclature section. 
BM-D-13-01063 - Rev. 2

Manuscript (unmarked copy) - $21 / 22$

Table 4 -Mean and range ([min ; max $] ; \pm \mathrm{SD})$ values of the deviations (in degrees) between two local frame definitions for the long fingers and the Radius (17 segments) and for the thumb (4 segments).

\begin{tabular}{|c|c|c|c|c|c|c|}
\hline & \multicolumn{3}{|c|}{ Long fingers and Radius } & \multicolumn{3}{|c|}{ Thumb } \\
\hline & Land. / Ref & Func. / Ref & Land. / Func. & Land. / Ref & Func. / Ref & Land. / Func. \\
\hline \multicolumn{7}{|l|}{$\Delta_{\text {tot }}\left({ }^{\circ}\right)$} \\
\hline [Min ; Max] & {$[6.5 ; 22.4]$} & {$[4.9 ; 28.1]$} & {$[14.9 ; 36.9]$} & {$[58.9 ; 68.2$} & {$[45.0 ; 70.8]$} & {$[14.1 ; 34.9]$} \\
\hline Mean. \pm SD & $14.4 \pm 4.1$ & $16.5 \pm 7.9$ & $22.0 \pm 6.6$ & $62.9 \pm 3.9$ & $53.7 \pm 12.2$ & $24.5 \pm 8.5$ \\
\hline \multicolumn{7}{|l|}{$\Delta_{X}\left({ }^{\circ}\right)$} \\
\hline [Min ; Max] & {$[0.1 ; 14.7]$} & {$[4.9 ; 27.9]$} & {$[4.9 ; 32.5]$} & {$[57.9 ; 60.3]$} & {$[44.8 ; 70.2]$} & {$[12.7 ; 19.1]$} \\
\hline Mean. \pm SD & $5.4 \pm 4.9$ & $16.5 \pm 7.9$ & $16.6 \pm 8.2$ & $58.8 \pm 1.1$ & $53.0 \pm 12.0$ & $15.4 \pm 3.0$ \\
\hline \multicolumn{7}{|l|}{$\Delta_{y}\left({ }^{\circ}\right)$} \\
\hline$[\operatorname{Min} ; \operatorname{Max}]$ & {$[6.5 ; 22.4]$} & {$[0.1 ; 18.9]$} & {$[9.4 ; 25.9]$} & {$[8.2 ; 44.4]$} & {$[10.6 ; 41.5]$} & {$[9.5 ; 24.5]$} \\
\hline Mean. \pm SD & $14.4 \pm 4.1$ & $8.5 \pm 5.8$ & $16.8 \pm 4.8$ & $21.8 \pm 16.2$ & $19.1 \pm 15.0$ & $18.3 \pm 6.5$ \\
\hline \multicolumn{7}{|l|}{$\Delta_{z}\left({ }^{\circ}\right)$} \\
\hline [Min ; Max] & {$[4.1 ; 22.4]$} & {$[0.5 ; 25.8]$} & {$[7.5 ; 31.1]$} & {$[58.2 ; 62.9]$} & {$[35.3 ; 69.6]$} & {$[12.2 ; 39.5]$} \\
\hline Mean. \pm SD & $12.4 \pm 4.7$ & $12.5 \pm 8.8$ & $18.7 \pm 7.2$ & $60.6 \pm 2.0$ & $48.1 \pm 14.9$ & $24.4 \pm 11.4$ \\
\hline
\end{tabular}

Table 5 - Mean and range ([min; max $] ; \pm \mathrm{SD})$ of the differences (degrees) between the amplitudes of Cardan angles estimated with the three definitions during the finger-flexion and the ball-grasping test motions. Range, mean and standard values computed among the long fingers' joints (4 metacarpophalangeal, 4 proximal interphalangeal and 4 distal interphalangeal joints).

\begin{tabular}{|c|c|c|c|c|c|c|}
\hline & \multicolumn{3}{|c|}{ Flexion motion } & \multicolumn{3}{|c|}{ Ball-grasping motion } \\
\hline 2 & Land. / Ref. & Func. / Ref. & Land. / Func. & Land. / Ref. & Func. / Ref. & Land. / Func. \\
\hline \multicolumn{7}{|l|}{ Flexion-extension } \\
\hline [Min ; Max] & {$[0.1 ; 4.2]$} & {$[0.1 ; 7.0]$} & {$[0.4 ; 5.6]$} & {$[0.0 ; 1.6]$} & {$[0.1 ; 3.1]$} & {$[0.2 ; 3.6]$} \\
\hline Mean \pm SD & $1.2 \pm 1.3$ & $2.3 \pm 2.1$ & $1.9 \pm 1.5$ & $0.6 \pm 0.5$ & $1.6 \pm 1.1$ & $1.5 \pm 0.9$ \\
\hline \multicolumn{7}{|l|}{ Abduction-adduction } \\
\hline [Min ; Max] & {$[0.1 ; 8.2]$} & {$[0.2 ; 23.6]$} & {$[0.2 ; 21.2]$} & {$[0.0 ; 5.9]$} & {$[1.2 ; 10.3]$} & {$[1.4 ; 5.5]$} \\
\hline Mean \pm SD & $4.1 \pm 3.2$ & $10.7 \pm 9.1$ & $8.6 \pm 6.9$ & $1.6 \pm 1.6$ & $4.3 \pm 2.8$ & $3.6 \pm 1.5$ \\
\hline \multicolumn{7}{|l|}{ Pronation-supination } \\
\hline [Min ; Max] & {$[0.8 ; 12.7]$} & {$[0.3 ; 13.2]$} & {$[1.2 ; 18.8]$} & {$[0.6 ; 6.2]$} & {$[0.3 ; 6.3]$} & {$[0.1 ; 12.4]$} \\
\hline Mean \pm SD & $4.3 \pm 3.5$ & $5.3 \pm 3.3$ & $7.2 \pm 6.2$ & $2.1 \pm 1.7$ & $2.9 \pm 2.1$ & $3.0 \pm 3.5$ \\
\hline
\end{tabular}


BM-D-13-01063 - Rev. 2

Manuscript (unmarked copy) - 22 / 22

\section{FIGURE LEGENDS}

Figure $1-$ A: Experimental setup showing a subject's hand apposed on the specially designed reference board prior to marker equipment. The bony landmarks were marked by dots prior to marker placement. - B: The marker set used to record kinematic data of the forearm and the five fingers.

Figure 2 - Local frames obtained with the three definitions for several segments and expressed in the Reference frames. Solid black lines represent Reference frames, solid grey lines represent Landmark frames and dashed black lines represent Functional frames. $\boldsymbol{x}$ axes are indicated by filled circles and $\boldsymbol{y}$ axes by blank circles.

Figure 3 - Representative results of the Cardan angles obtained with the three local frame definitions during the flexion-extension test motion. MCP4, PIP4, DIP4 refers to the ring finger metacarpophalangeal, proximal interphalangeal and distal interphalangeal joints.

Figure 4 - Representative results of the Cardan angles obtained with the three local frame definitions during the ball-grasping test motion. MCP3 and TMC refer to the middle finger metacarpophalangeal joint and the thumb trapeziometacarpal joint. The first 50 frames correspond to the reaching movement and the 50 others to the grasping posture.

Figure 5 - Illustration of the differences that could be observed between local axes of the proximal phalanx of the index finger computed with different methods for a patient with ulnar deviations (A) and for another with a boutonnière deformity (B). Black vectors $\left(\boldsymbol{x}_{\mathrm{R}}, \boldsymbol{y}_{\mathrm{R}}, \boldsymbol{z}_{\mathrm{R}}\right)$ represent the Reference definition and grey vectors $\left(y_{\mathrm{L}}\right)$ represent the Landmark definition. 


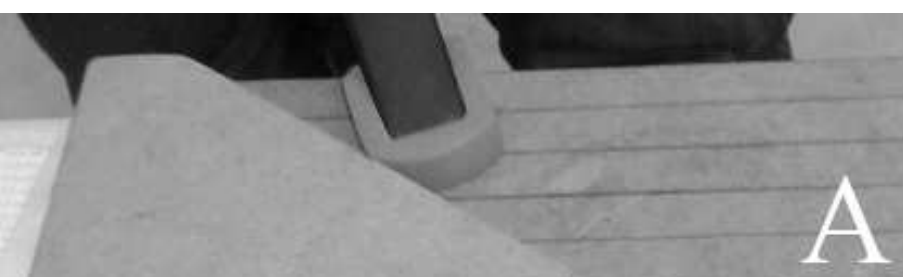

A
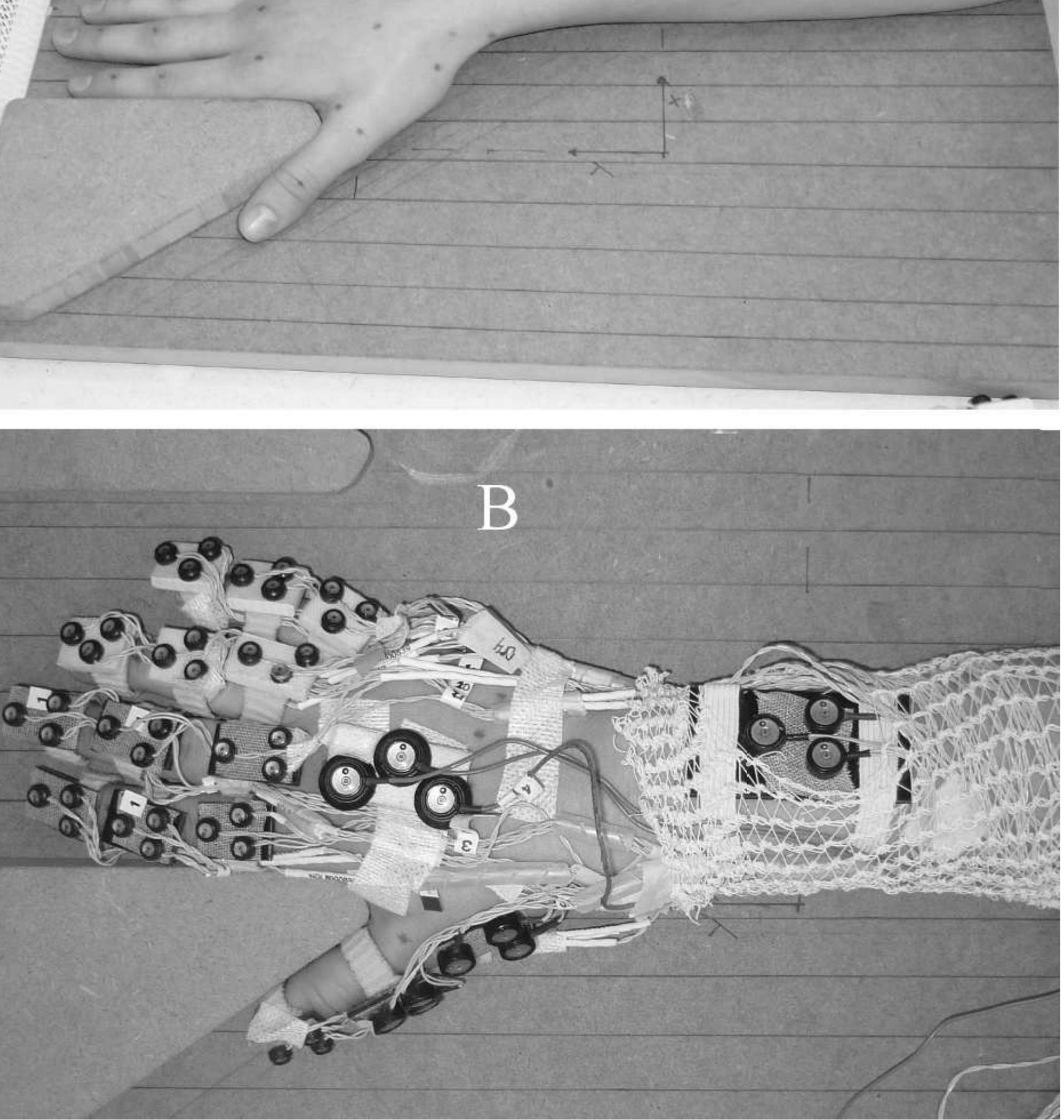

\section{B}




\begin{tabular}{|c|c|c|}
\hline \multicolumn{3}{|c|}{ Local frames } \\
\hline - Reference & L Landmark & -m Functional \\
\hline
\end{tabular}

Radius

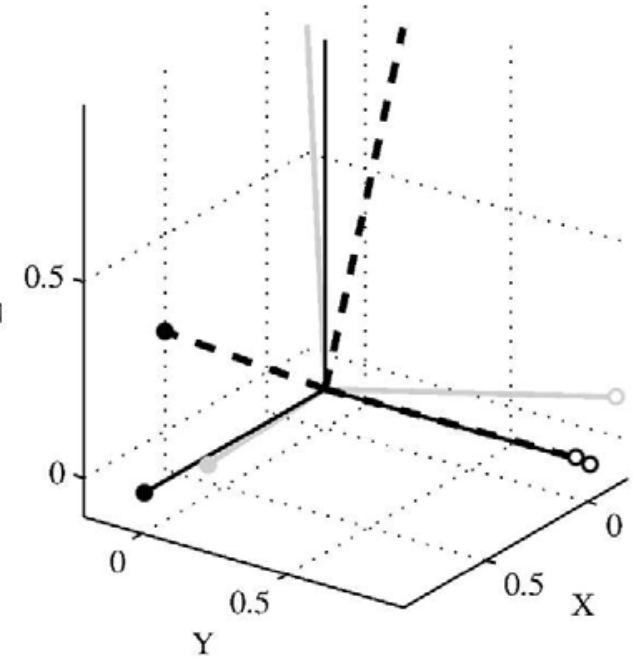

MC3

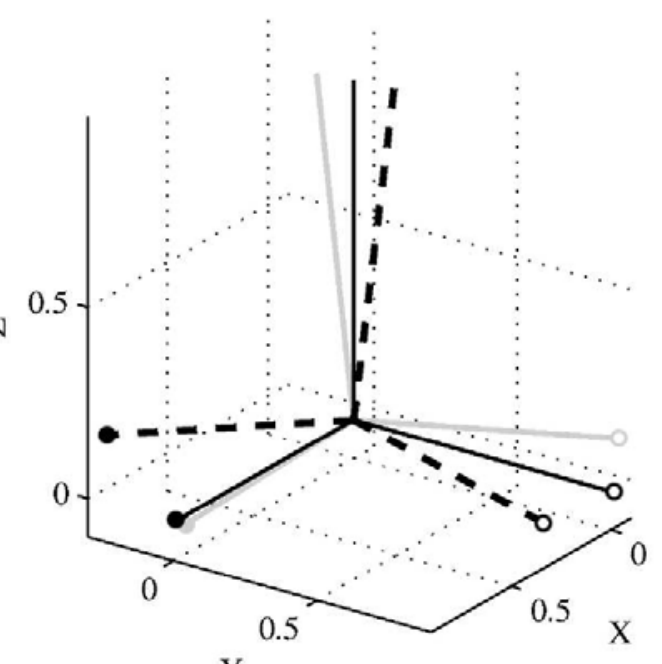

Trapezium

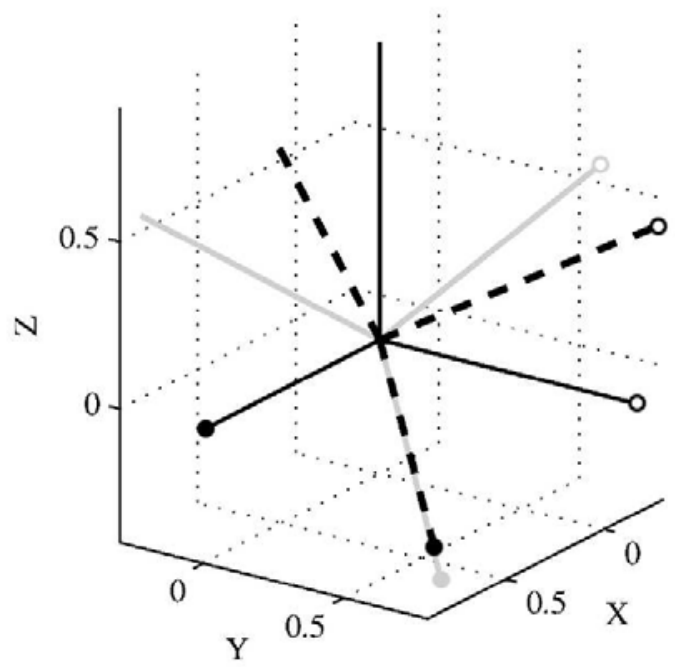

PP3

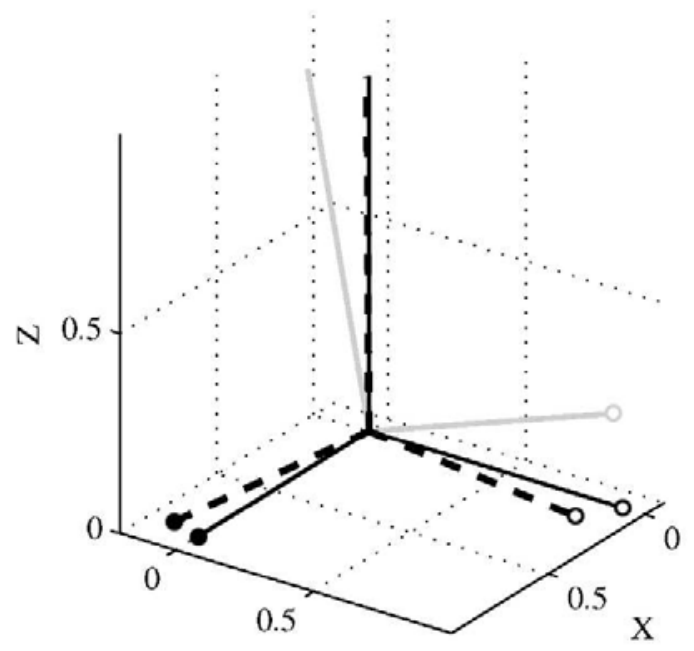

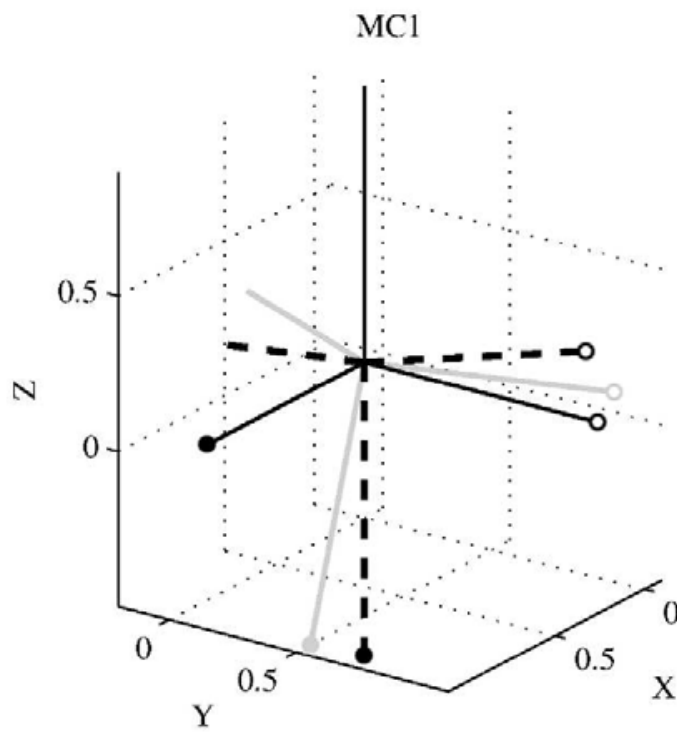

MP3

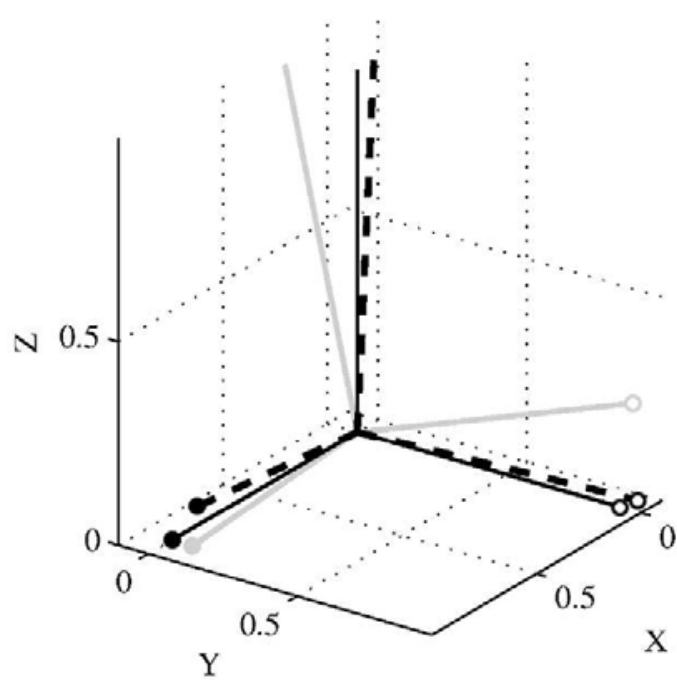


MCP3

Flexion-Extension

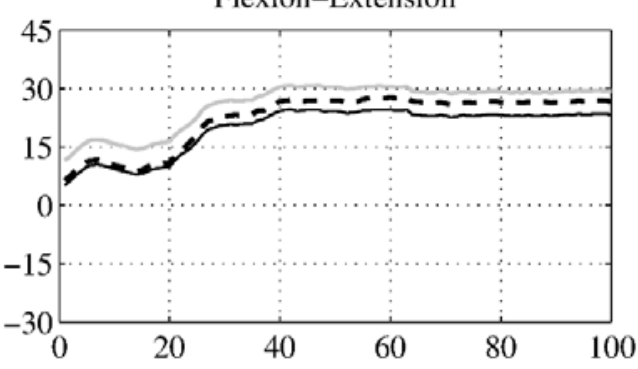

Abduction-Adduction

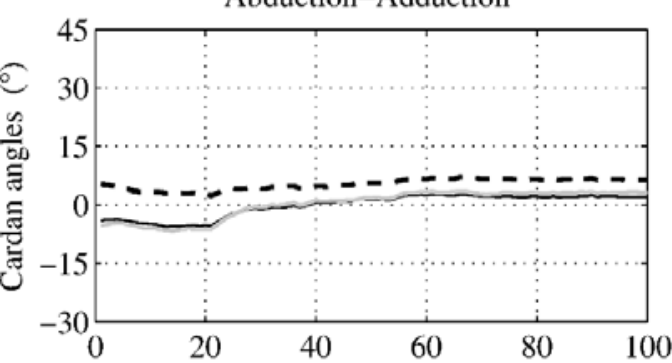

Pronation-Supination

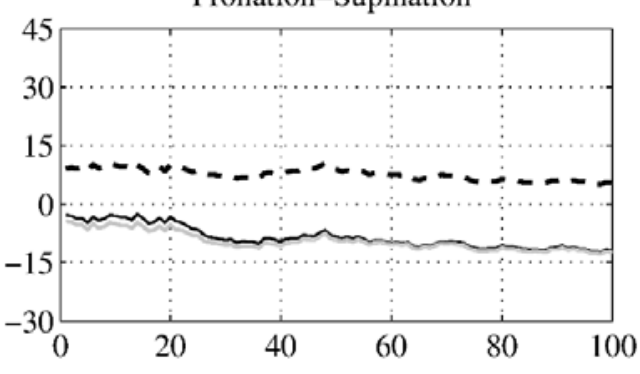

\section{Reference} - Landmark

TMC

Flexion-Extension

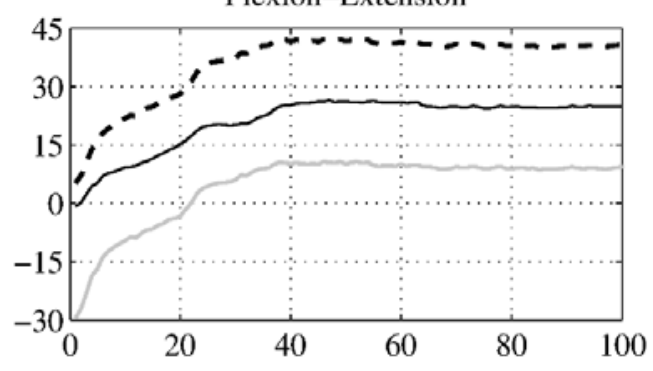

Abduction-Adduction

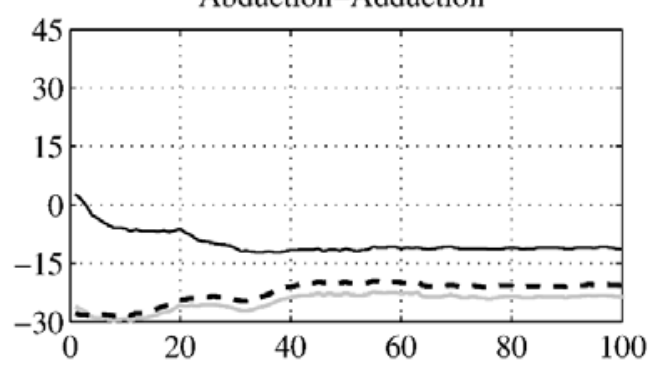

Pronation-Supination

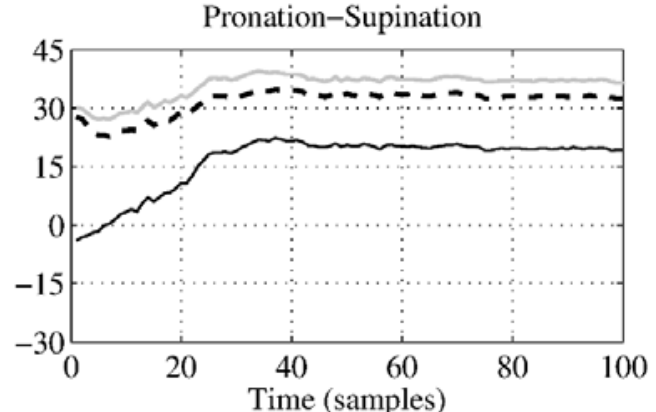

Wrist

Flexion-Extension

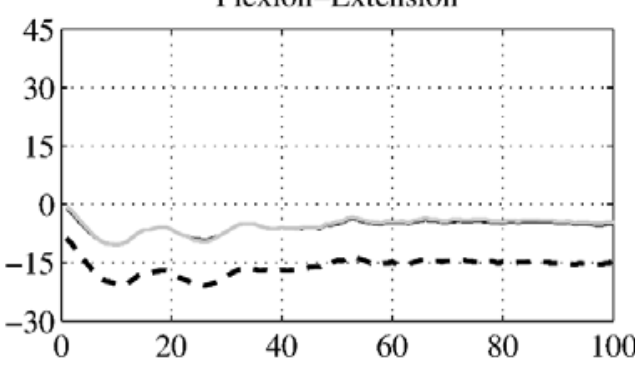

Abduction-Adduction

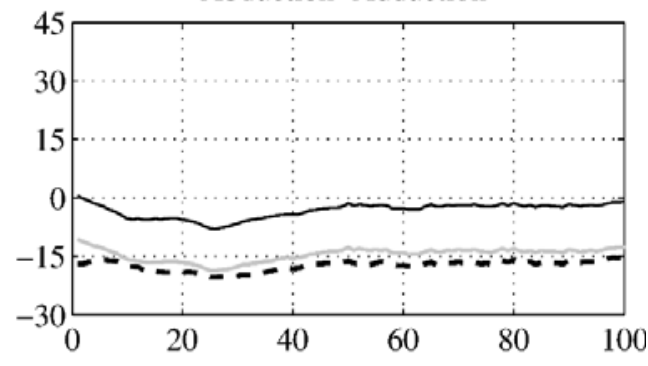

Pronation-Supination

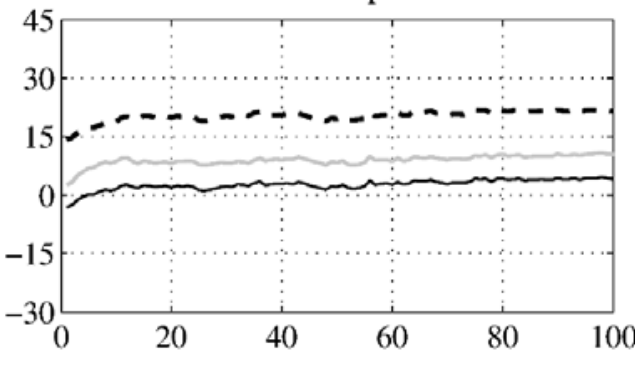


A

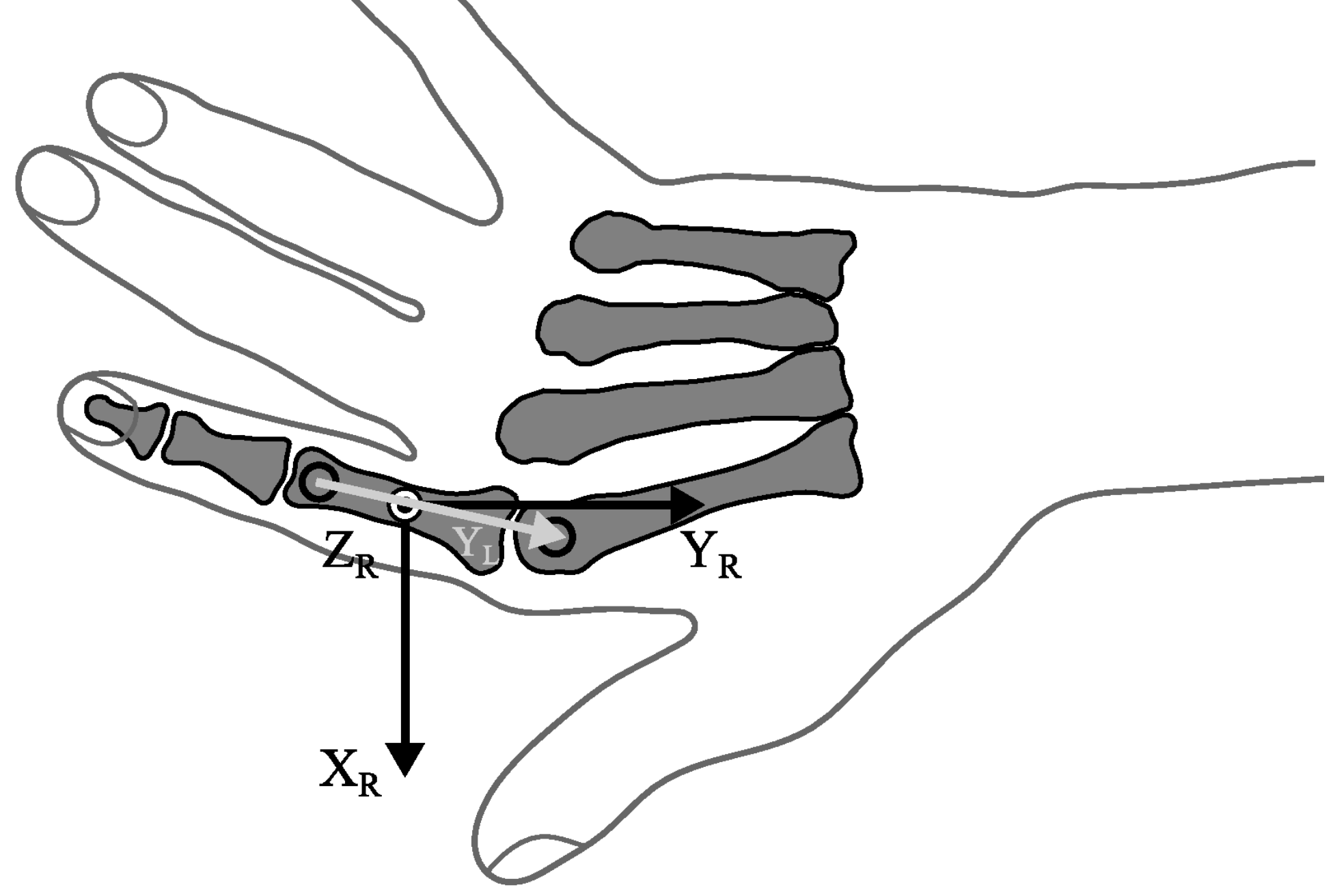

B
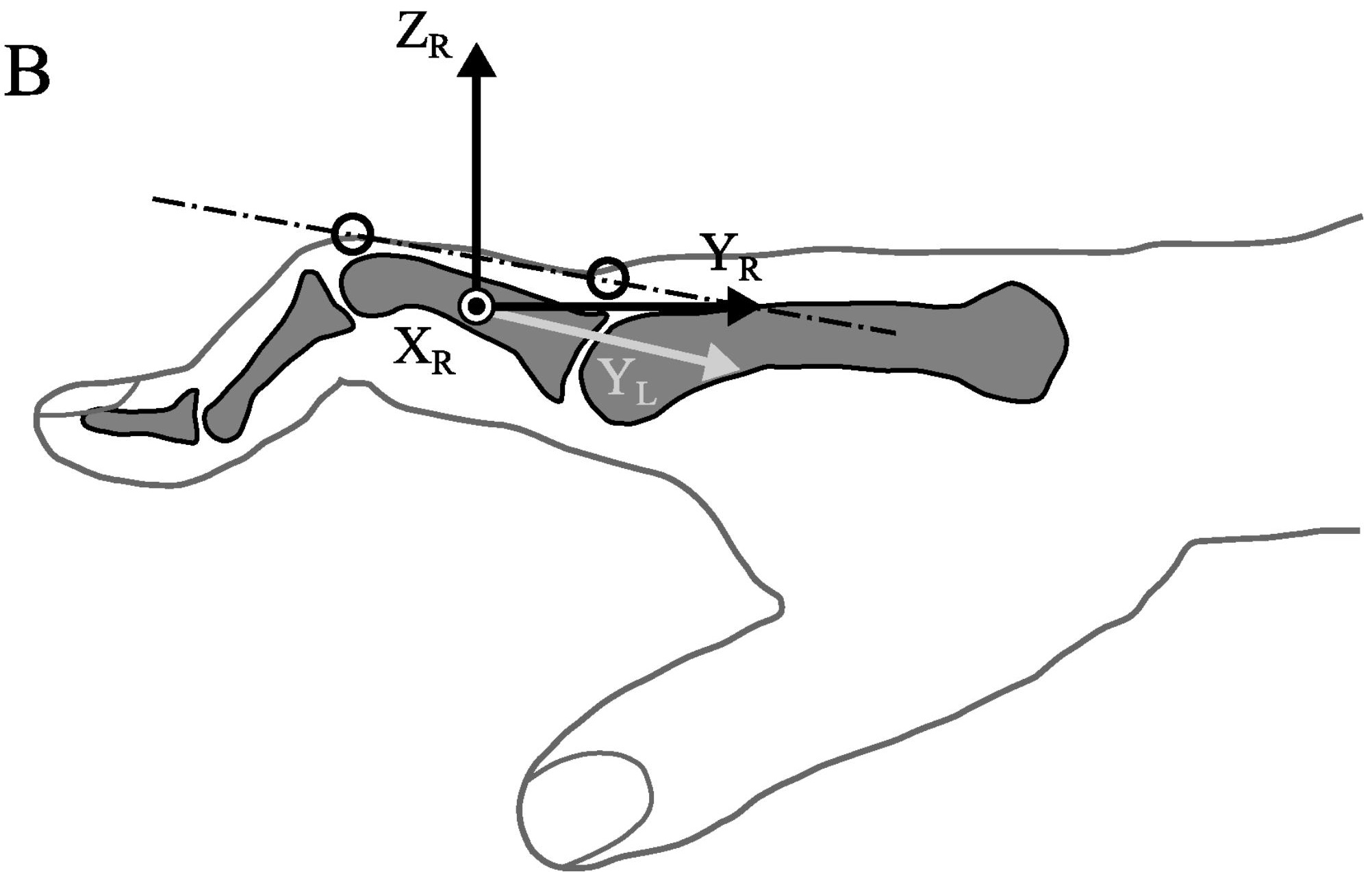